\title{
Covariant Time-Frequency Representations Through Unitary Equivalence
}

\author{
Richard G. Baraniuk, Member, IEEE
}

\begin{abstract}
We propose a straightforward characterization of all quadratic time-frequency representations covariant to an important class of unitary signal transforms (namely, those having two continuous-valued parameters and an underlying group structure). Thanks to a fundamental theorem from the theory of Lie groups, we can describe these representations simply in terms of unitary transformations of the well-known Cohen's and affine classes.
\end{abstract}

\section{INTRODUCTION}

Q UADRATIC time-frequency representations (TFR's) have found wide application in problems requiring timevarying spectral analysis [1], [2]. Since the distribution of signal energy jointly over time and frequency coordinates does not have a unique representation, there exist many different TFR's and many different ways to obtain them. Up to the present, two classes of TFR's have predominated: Cohen's class [1] and the affine class [25]. Cohen's class TFR's are covariant to time and frequency shifts in the signal, whereas affine class TFR's are covariant to time shifts and scale changes in the signal.

Since time shifts, frequency shifts, and scale changes are not the only important signal transformations occurring in nature, several new TFR classes matching different transformations have been proposed recently. The TFR's of the hyperbolic class [6] are covariant to "hyperbolic time shifts" and scale changes, whereas the TFR's of the power classes [7] are covariant to "power time shifts" and scale changes. Unitarily transformed Cohen's and affine classes furnish even more TFR's [8], [9]. While extremely simple both in concept and in application, the unitary equivalence or "warping" procedure that generates these TFR's leads at once to an infinite number of new TFR classes covariant to a broad class of signal transformations. Coping with this veritable explosion of new TFR classes demands a comprehensive theory for time-frequency analysis.

In this paper, we propose a simple characterization of covariant TFR's based on the theory of unitary equivalence. The sufficiency of unitary equivalence for this task comes as somewhat of a surprise, since this method proves more powerful than previously surmised.

Manuscript received June 13, 1995. This work was supported by the National Science Foundation under Grant MIP-9457438, by the Office of Naval Research under Grant N00014-95-1-0849, and by the Texas Advanced Technology Program under Grant TX-ATP 003604-002. The associate editor coordinating the review of this letter and approving it for publication was Prof. D. L. Jones.

The author is with the Department of Electrical and Computer Engineering, Rice University, Houston, TX 77251-1892 USA.

Publisher Item Identifier S 1070-9908(96)02744-7.

\section{Cohen's Class and the AfFine Class}

We denote the TFR of a signal $s \in L^{2}(\mathbf{R})$ using the operator notation $(\mathbf{P} s)(t, f)$, where $\mathbf{P}: L^{2}(\mathbf{R}) \rightarrow L^{2}\left(\mathbf{R}^{2}\right)$ is the TFR mapping, and $t$ and $f$ are the time and frequency coordinates, respectively. We will emphasize only quadratic TFR's in this paper.

Each TFR in Cohen's class [1], [2] can be expressed as

$$
(\mathbf{C} s)(t, f)=\iint(\mathbf{A} s)(\theta, \tau) \phi(\theta, \tau) e^{-j 2 \pi(\theta t+\tau f)} d \theta d \tau
$$

in terms of the narrowband ambiguity function of the signal $(\mathbf{A} s)(\theta, \tau) \equiv \int s\left(t+\frac{\tau}{2}\right) s^{*}\left(t-\frac{\tau}{2}\right) e^{j 2 \pi \theta t} d t$ and a kernel function $\phi(\theta, \tau)$. TFR's generated by fixed kernels are covariant to the time shift operator $\left(\mathbf{T}_{n} s\right)(x) \equiv s(x-n)$ and the frequency shift operator $\left(\mathbf{F}_{m} s\right)(x) \equiv e^{j 2 \pi m x} s(x)$

$$
\left(\mathbf{C F}_{m} \mathbf{T}_{n} s\right)(t, f)=(\mathbf{C} s)(t-n, f-m)
$$

with $m, n \in \mathbb{R}$. Conversely, all quadratic TFR's covariant in this way must belong to Cohen's class [2]. Covariance by translation is natural for Cohen's class TFR's, because $\mathbf{T}$ and F comprise the heart of the unitary representation on $L^{2}(\mathbb{R})$ of the Weyl-Heisenberg group, with

$$
\left(\mathbf{F}_{m_{1}} \mathbf{T}_{n_{1}}\right)\left(\mathbf{F}_{m_{2}} \mathbf{T}_{n_{2}}\right)=e^{-j 2 \pi m_{2} n_{1}} \mathbf{F}_{m_{1}+m_{2}} \mathbf{T}_{n_{1}+n 2} .
$$

(See [5], [10], and [11] for more details on the role of group theory in time-frequency analysis.)

Each TFR in the affine class [2]-[5] can be expressed as

$$
(\mathbf{Q} s)(t, f)=\iint(\mathbf{A} s)(\theta, \tau) \psi(\theta / f, f \tau) e^{-j 2 \pi(\theta t+\tau f)} d \theta d \tau
$$

with kernel $\psi(\theta, \tau)$. TFR's generated by fixed kernels are covariant to the time shift operator and the scale change operator $\left(\mathbf{D}_{d} s\right)(x) \equiv|d|^{-1 / 2} s(x / d)$

$$
\left(\mathbf{Q T}_{n} \mathbf{D}_{d} s\right)(t, f)=(\mathbf{Q} s)\left(\frac{t-n}{d}, d f\right) .
$$

Conversely, all quadratic TFR's covariant in this way must belong to the affine class [2], [4], [5]. Affine covariance is natural for these TFR's, because $T$ and $\mathbf{D}$ comprise the unitary representation on $L^{2}(\mathbb{R})$ of the affine group, with

$$
\left(\mathbf{T}_{n_{1}} \mathbf{D}_{d_{1}}\right)\left(\mathbf{T}_{n_{2}} \mathbf{D}_{d_{2}}\right)=\mathbf{T}_{n_{1}+d_{1} n_{2}} \mathbf{D}_{d_{1} d_{2}} .
$$




\section{UNITARILY EQUiVALENT \\ TIME-FREQUENCY REPRESENTATIONS}

To match signal transformations different from time shifts, frequency shifts, and scale changes, new classes of TFR's have been developed, including the hyperbolic class of TFR's covariant to scale changes and "hyperbolic time shifts" [6] and the power classes of TFR's covariant to scale changes and "chirp time shifts" [7]. While both of these classes can be derived from first principles, they can also be obtained directly by transforming Cohen's class and the affine class.

In particular, each hyperbolic class TFR can be expressed as VCU, where $\mathbf{C}$ is a Cohen's class TFR, $\mathbf{U}: L^{2}(\mathbb{R}) \rightarrow L^{2}(\mathbb{R})$ is a unitary signal transformation, and $\mathbf{V}: L^{2}\left(\mathbb{R}^{2}\right) \rightarrow L^{2}\left(\mathbb{R}^{2}\right)$ is a unitary change of variables [6]. Each power class TFR can be similarly expressed as VQU, where $Q$ is an affine class TFR [7]. (See [6], [7], and [9] for the exact form of the transformations $U$ and $V$.) The primary advantage of this transformation-based derivation is its conceptual and computational simplicity: To compute a hyperbolic or power class TFR, we simply preprocess the signal by the unitary transform U, compute a Cohen's class TFR $\mathbf{C}$ or affine class TFR $Q$ of the transformed signal, and then warp the axes of the resulting distribution by $\mathrm{V}$.

Transformation of the Cohen's and affine classes does not have to stop with the hyperbolic and power classes. By varying $\mathbf{U}$ and $\mathbf{V}$, we can generate an infinite number of transformed Cohen's and affine classes. We now summarize the salient features of the resulting theory of unitarily equivalent TFR's developed in detail in [9].

Transformed Cohen's and affine class distributions are covariant, not to time and frequency shifts and scale changes, but to the unitarily equivalent operators

$$
\widetilde{\mathbf{T}}_{n}=\mathbf{U}^{-1} \mathbf{T}_{n} \mathbf{U}, \quad \widetilde{\mathbf{F}}_{m}=\mathbf{U}^{-1} \mathbf{F}_{m} \mathbf{U}, \quad \widetilde{\mathbf{D}}_{d}=\mathbf{U}^{-1} \mathbf{D}_{d} \mathbf{U} .
$$

To see this, ignore $\mathbf{V}$ for the moment, and note that

$$
\begin{aligned}
& \left(\mathbf{C U} \widetilde{\mathbf{F}}_{m} \widetilde{\mathbf{T}}_{n} s\right)(a, b)=(\mathbf{C U} s)(a-n, b-m) \\
& \left(\mathbf{Q U} \widetilde{\mathbf{T}}_{n} \widetilde{\mathbf{D}}_{d} s\right)(a, b)=(\mathbf{Q U} s)\left(\frac{a-n}{d}, d b\right) .
\end{aligned}
$$

The preprocessed distributions $\mathrm{CU}$ and $\mathrm{QU}$ maintain the same translation and affine covariances exhibited by the distributions from which they are derived (compare (8) with (2) and (9) with (5)), because the operator pairs $\widetilde{\mathbf{F}} \widetilde{\mathbf{T}}$ and $\widetilde{\mathbf{T}} \widetilde{\mathbf{D}}$ remain unitary representations of the Weyl-Heisenberg and affine groups, respectively. Thus, transformed Cohen's and affine classes are unitarily equivalent to the original Cohen's and affine classes.

While the coordinates $(a, b)$ of the preprocessed distributions CU and QU do not correspond to time and frequency (in fact, they correspond to the physical quantities associated with the operators $\widetilde{\mathbf{T}}$ and $\widetilde{\mathbf{F}}$ ), the postprocessing transformation $\mathbf{V}$ can warp $(a, b)$ to new coordinates providing correct time-frequency localization [9]. Given a fixed $U$, the procedure to determine the corresponding $\mathrm{V}$ is straightforward: We simply warp the axes of the distributions by functions $A(t, f)$ and $B(t, f)$ that describe the group delay and instantaneous frequency of the transformed eigenfunctions $\mathbf{U}^{-1} \delta(x-a)$ and $\mathbf{U}^{-1} e^{j 2 \pi b x}$ of $\widetilde{\mathbf{F}}$ and $\widetilde{\mathbf{T}}$, respectively. ${ }^{1}$ For transformed Cohen's class distributions, we set

$$
(\mathbf{V C U} s)(t, f)=(\mathbf{C U} s)[A(t, f), B(t, f)] .
$$

The affine class case is similar.

The unitarily equivalent TFR's VCU and VQU remain covariant to the operators $\widetilde{\mathbf{F}} \widetilde{\mathbf{T}}$ and $\widetilde{\mathbf{T}} \widetilde{\mathbf{D}}$, respectively, although $\mathrm{V}$ warps the group actions (8), (9) along the group delay and instantaneous frequency curves of the transformed eigenfunctions [9].

The simplicity of the unitary equivalence principle makes the study of the properties of unitarily equivalent TFR classes essentially trivial, since the attributes of any transformed class can be obtained immediately from those of Cohen's class or the affine class by a simple translation procedure. (We simply replace $s$ by $\mathbf{U} s$ throughout and warp the axes.) This translation has been performed in detail in [9].

\section{A UNIFIED THEORY FOR COVARIANT TIME-FREQUENCY REPRESENTATIONS}

Unitary equivalence provides a simple means for developing an infinite number of different TFR classes. However, it is not $a$ priori obvious that this theory encompasses all possible covariant TFR's. For instance, unitarily equivalent TFR's are bound (within warping) to the Weyl-Heisenberg group and affine group covariances they inherit from Cohen's class and the affine class. (In [8] and [9], this was viewed as a limitation of the theory.) A comprehensive theory of covariant TFR's thus seems a worthy goal.

\section{A. Covariant Time-Frequency Representations}

Our formulation will characterize TFR's covariant to a class of two-parameter unitary signal transformations $\mathbf{G}_{(p, q)}$ that generalize the time-frequency shift and time-scale change operators that are so natural for Cohen's class and affine class TFR's. Physical considerations (invertibility, composition, etc.) dictate that each of these transformations be a unitary group representation with group law "•" [5], [10], [11]

$$
\mathbf{G}_{\left(p_{1}, q_{1}\right)} \mathbf{G}_{\left(p_{2}, q_{2}\right)}=\mathbf{G}_{\left(p_{1}, q_{1}\right) \bullet\left(p_{2}, q_{2}\right)} \text {. }
$$

We say a TFR $(\mathbf{P} s)(t, f)$ is covariant to $\mathbf{G}_{(p, q)}$ if

$$
\left(\mathbf{P G}_{(p, q)} s\right)(t, f)=(\mathbf{P} s)(t, f) \diamond(p, q),
$$

where " $\circ$ " is the representation of $\mathbf{G}_{(p, q)}$ on the time-frequency plane (specifically, the coadjoint representation [5], [10], [11]). Note that the group property (11) of $\mathbf{G}_{(p, q)}$ immediately yields that

$$
\left(\mathbf{P G}_{\left(p_{1}, q_{1}\right)} \mathbf{G}_{\left(p_{2}, q_{2}\right)} s\right)(t, f)=(\mathbf{P} s)(t, f) \diamond\left[\left(p_{1}, q_{1}\right) \bullet\left(p_{2}, q_{2}\right)\right] \text {. }
$$

A similar (and equivalent) approach to covariance has been developed independently by Hlawatsch and Bölcskei in [12] and [13]. In their terminology, $\mathrm{G}$ is a time-frequency displacement operator with " $\diamond$ " the associated displacement function.

\footnotetext{
${ }^{1}$ The group delay of $\mathbf{U}^{-1} \delta(x-a)$ lies along the curve $a=A(t, f)$. The instantaneous frequency of $\mathbf{U}^{-1} e^{j 2 \pi b x}$ lies along the curve $b=B(t, f)$ [9].
} 
Examples of displacement operators and functions include the following.

1) $\mathbf{G}_{(m, n)}=\mathbf{F}_{m} \mathbf{T}_{n}$ and (2) for Cohen's class TFR's. ${ }^{2}$

2) $\mathbf{G}_{(n, d)}=\mathbf{T}_{n} \mathbf{D}_{d}$ and (5) for affine class TFR's.

3) $\mathbf{G}_{(m, n)}=\widetilde{\mathbf{F}}_{m} \widetilde{\mathbf{T}}_{n}=\mathbf{U}^{-1} \mathbf{F}_{m} \mathbf{T}_{n} \mathbf{U}$ and a version of (2) warped by $A(t, f), B(t, f)$ for unitarily equivalent Cohen's class TFR's.

4) $\mathbf{G}(n, d)=\mathbf{U}^{-1} \mathbf{T}_{n} \mathbf{D}_{d} \mathbf{U}$ and a version of (5) warped by $A(t, f), B(t, f)$ for unitarily equivalent affine class TFR's.

\section{B. A Simple Unified Theory}

Unitary equivalence generates TFR's covariant to an infinite number ofdifferent two-parameter displacement operators $\mathbf{G}$. What we now show is that there exist no covariant TFR's: beyond these. In other words, the simple unitary equivalence procedure described in Section III proves sufficient for characterizing all covariant TFR's.

The key realization is the following: Since the displacement operator $\mathbf{G}$ determining the covariance properties of a TFR class is constrained by (11) to be a unitary group representation, it is clear that the classification of all covariant TFR classes is equivalent to the classification of all two-parameter Lie groups (within a phase) that can act on the signal space $L^{2}(\mathbb{R})$. It has been recently pointed out [11] that there exist only two such Lie groups: the Weyl-Heisenberg group leading to displacement operators of the form $\mathbf{G}=\mathbf{U}^{-1} \mathbf{F T U}$ and the affine group leading to displacement operators of the form $\mathbf{G}=\mathbf{U}^{-1} \mathbf{T D U}$. Since the TFR classes covariant to these displacements correspond to unitarily equivalent Cohen's and affine classes, we have the following fundamental result.

Theorem: All quadratric TFR's that are covariant in the sense of Section IV-A can be represented in the form VCU or VQU with $\mathbf{C}$ a Cohen's class TFR, $\mathbf{Q}$ an affine class TFR, $\mathrm{U}$ a unitary signal transformation, and $\mathrm{V}$ a 2-D coordinate transformation as described in Section III.

\section{DisCuSSION AND CONCLUSIONS}

An observation regarding the dearth of two-parameter covariances has lead us to an extremely simple characterization of all covariant quadratic TFR classes as unitarily transformed Cohen's or affine classes. The "shortcut" of unitary equivalence gives this theory several advantages. First, we see

\footnotetext{
${ }^{2}$ The Weyl-Heisenberg group actually has three, and not two, parameters; therefore, the displacement operator $\mathbf{G}_{(n, m)}=\mathbf{F}_{m} \mathbf{T}_{n}$ does not strictly obey (11). However, since the desired group $\mathbb{R}^{2}$ of translations in the time-frequency plane does not have a representation on the space $L^{2}(\mathbb{R})$, we are forced to employ the Weyl-Heisenberg group in signal processing applications. Fortunately, the third parameter plays the role of a phase and can be ignored.
}

immediately that the set of all two-parameter time-frequency displacement operators inducing covariant TFR's is limited to operators of the form $\mathbf{U}^{-1} \mathbf{F T U}$ or $\mathbf{U}^{-1} \mathbf{T D U}$. Second, since each covariant TFR class corresponds directly to either Cohen's class or the affine class, we can leverage our years of experience with these classes into new contexts with no additional effort.

It is straightforward to demonstrate the equivalence of our theory to that of Hlawatsch and Bölcskei [12], [13], since the axioms they impose on a time-frequency displacement operator constrain it to be (within a phase) a unitary representation of a two-parameter Lie group. The prime advantage of the present theory is its ease of use; in contrast, the Hlawatsch-Bölcskei construction appears quite complicated. Thus, despite its striking simplicity, the power and generality of unitary equivalent time-frequency analysis should not be underestimated.

\section{ACKNOWLEDGMENT}

The author wishes to thank P. Gonçalvés for reading and discussing the manuscript.

\section{REFERENCES}

[1] L. Cohen, Time-Frequency Analysis. Englewood Cliffs, NJ: PrenticeHall, 1995.

[2] P. Flandrin, Temps-Fréquence. Paris: Hermes, 1993.

[3] T. Posch, "Wavelet transforms and time-frequency distributions," in Proc. SPIE Int. Soc. Opt. Eng., vol. 1152, 1988, pp. 477-482.

[4] O. Rioul and P. Flandrin, "Time-scale energy distributions: A general class extending wavelet transforms," IEEE Trans. Signal Processing, vol. 40, pp. 1746-1757, July 1992.

[5] J. Bertrand and P. Bertrand, "A class of affine Wigner functions with extended covariance properties," J. Math. Phys., vol. 33, pp. 2515-2527, July 1992.

[6] A. Papandreou, F. Hlawatsch, and G. F. Boudreaux-Bartels, "The hyperbolic class of quadratic time-frequency representations. Part I: Constant- $Q$ warping, the hyperbolic paradigm, properties, and members," IEEE Trans. Signal Processing, vol. 41, pp. 3425-3444, Dec. 1993.

[7] F. Hlawatsch, A. Papandreou, and G. F. Boudreaux-Bartels, "The power classes of quadratic time-frequency representations: A generalization of the affine and hyperbolic classes," in Proc. 27th Asilomar Conf., Pacific Grove, CA, 1993, pp. 1265-1270.

[8] R. G. Baraniuk and L. Cohen, "On joint distributions of arbitrary variables," IEEE Signal Processing Lett., vol. 2, pp. 10-12, Jan. 1995.

[9] R. G. Baraniuk and D. L. Jones, "Unitary equivalence: A new twist on signal processing," IEEE Trans. Signal Processing, vol. 43, Oct. 1995.

[10] R. G. Shenoy and T. W. Parks, "Wide-band ambiguity functions and affine Wigner distributions," Signal Processing, vol. 41, no. 3, pp. 339-363, 1995.

[11] R. G. Baraniuk, "Marginals vs. covariance in joint distribution theory," in Proc. IEEE Int. Conf. Acoust., Speech, Signal Processing, vol. 2, 1995, pp, 1021-1024.

[12] F. Hlawatsch and H. Bölcskei, "Unified theory of displacementcovariant time-frequency analysis," in Proc. IEEE Int. Symp. TimeFrequency Time-Scale Anal., Oct. 1994, pp. 524-527.

[13] F. Hlawatsch and H. Bölcskei, "Displacement-covariant time-frequency energy distributions," in Proc. IEEE Int. Conf. Acoust., Speech, Signal Processing, vol. 2, 1995, pp. 1025-1028. 